\section{Utilidad del estudio contrastado precoz del tracto digestivo superior después de cirugía bariátrica: ¿se justifica su uso rutinario?}

\author{
FRANCISCO J. MEDINA ${ }^{1}$, ANDRÉS MIRANDA-MERCHAK ${ }^{1, a}$, \\ ALONSO MARTÍNEZ ${ }^{1}$, FELIPE SÁNCHEZ ${ }^{1, a}$, \\ SEBASTIÁN BRAVO ${ }^{1, a}$, JUAN EDUARDO CONTRERAS ${ }^{2}$, \\ ISABEL ALLIENDE ${ }^{3, \mathrm{a}}$, ANDREA CANALS ${ }^{3}$
}

\section{Usefulness of upper gastrointestinal series to detect leaks in the early postoperative period of bariatric surgery}

Background: Postoperative leaks are the most undesirable complication of bariatric surgery and upper gastrointestinal (GI) series are routinely ordered to rule them out. Despite the published literature recommending against its routine use, it is still being customarily used in Chile. Aim: To examine the usefulness of routine upper GI series using water-soluble iodinated contrast media for the detection of early postoperative leaks in patients undergoing bariatric surgery. Material and Methods: A cohort of 328 patients subjected to bariatric surgery was followed from October 2012 to October 2013. Most of them underwent sleeve gastrectomy. Results: Upper GI series on the first postoperative day were ordered to 308 (94\%) patients. Postoperative leaks were observed in two patients, with an incidence of $0.6 \%$. The sensitivity for upper GI series detection of leak was $0 \%$ and the negative predictive value was 99\%. Conclusions: Routine upper GI series after bariatric surgery is not useful for the diagnosis of postoperative leak, given the low incidence of this complication and the low sensitivity of the technique.

(Rev Med Chile 2016; 144: 451-455)

Key words: Anastomotic Leak; Bariatric Surgery; Upper Gastrointestinal Tract.

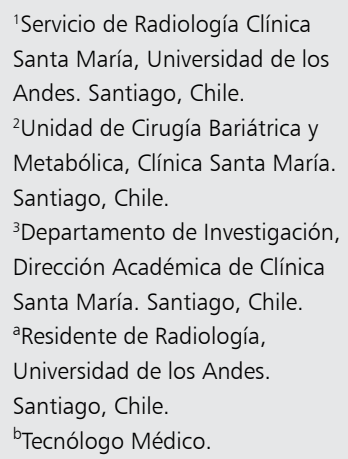

Recibido el 20 de junio de 2015, aceptado el 26 de diciembre de 2015.

Correspondencia a: Andrés Miranda-Merchak andresrobertomiranda@gmail.com
$\mathrm{E}$ l sobrepeso, la obesidad y su variedad mórbida han aumentado importantemente su prevalencia en Chile, alcanzando actualmente valores de $25,13 \%$ para obesidad (índice de masa corporal [IMC] sobre 30) y 2,3\% para obesidad mórbida (IMC sobre 40) según la Encuesta Nacional de Salud 2009-2010². En directa proporción con ello se ha experimentado un explosivo aumento de las cirugías bariátricas, con resultados que van más allá del solo objetivo de la reducción del peso corporal, como por ejemplo el control de la diabetes mellitus. Estos procedimientos quirúrgicos no están exentos de complicaciones, siendo la más temida la filtración postoperatoria ${ }^{2}$, por lo que el diagnóstico oportuno de ella es necesario para disminuir la morbimortalidad en estos pacientes ${ }^{3}$.

El estudio contrastado del tracto digestivo superior (ECTDS)-luego de la cirugía bariátrica laparoscópica- suele solicitarse de rutina para evaluar la presencia de filtración ${ }^{4}$. A pesar de la abundante evidencia que demuestra lo innecesario de este examen de rutina en el postoperatorio temprano (días 1 a 3$)^{3-9}$, y su carácter no costo-efectivo ${ }^{10}$, 
éste se suele seguir realizando en nuestro medio como norma ${ }^{11}$.

Este trabajo buscó determinar la utilidad del ECTDS de rutina, con medio de contraste yodado hidrosoluble, para la detección de filtración en el postoperatorio precoz en pacientes sometidos a bypass gástrico o gastrectomía vertical en manga por vía laparoscópica.

\section{Pacientes y Métodos}

Se realizó un estudio observacional, tipo cohorte prospectiva a partir de los casos operados en la Clínica Santa María con técnicas de bypass gástrico o de gastrectomía vertical en manga por laparoscopía, entre los meses de octubre de 2012 y octubre de 2013. En este período, 336 pacientes fueron operados por equipos quirúrgicos que emplean el ECTDS de rutina.

La técnica quirúrgica fue estándar entre los cirujanos y se realizó verificación de filtración intraoperatoria con azul de metileno en todos los casos. Luego, en el primer día de postoperatorio, los pacientes se sometieron al ECTDS con medio de contraste yodado hidrosoluble, utilizando para ello IOHEXOL (Omnipaque 300; GE Healthcare, Princeton, NJ). El examen fue realizado por un radiólogo staffen un equipo de fluoroscopia (Axiom Luminos TF, Siemens AG, Alemania).

La presencia de filtración se definió como la positividad del ECTDS en el primer día postoperatorio, o su evidencia en el seguimiento clínico posterior (por ejemplo, reintervención).

Las variables clínicas, demográficas y radiológicas pre y postoperatorias fueron obtenidas del
Registro Clínico Electrónico de la Clínica Santa María.

Se construyó una tabla de contingencia $2 \times 2$ para determinar sensibilidad, especificidad, valor predictivo positivo y valor predictivo negativo del ECTDS.

\section{Resultados}

Entre los meses de octubre de 2012 y octubre de 2013 se ingresaron 336 cirugías bariátricas laparoscópicas al protocolo. De éstas, 292 fueron manga gástrica $(87,7 \%)$ y 36 bypass gástrico $(12,3 \%)$ (Tabla 1). Existió una preponderancia de género al haber más mujeres (200) que hombres (128) realizándose cirugía de obesidad. El IMC preoperatorio estuvo dentro del rango de indicación de cirugía para los pacientes de esta cohorte. La estadía hospitalaria promedio fue de 3,2 días, siendo concordante con la realización del ECTDS dentro del primer día postoperatorio. El seguimiento a 3 meses de esta cohorte alcanzó 96,3\%.

Del total de 328 pacientes, a 308 se les solicitó estudio ECTDS en el primer día de postoperatorio $(93,9 \%)$. La ocurrencia de filtración postoperatoria se constató en 2 pacientes, correspondiendo a una incidencia de $0,6 \%$. De éstos, uno correspondió a una manga gástrica (incidencia $0,3 \%$ ) y el otro a un bypass gástrico (incidencia 2,7\%) (Tabla 1$)$. A 20 pacientes $(6,1 \%$ del total) no les fue solicitado el examen, mayoritariamente por decisión del cirujano tratante, no obstante, todos estos pacientes evolucionaron sin complicaciones.

El primer paciente con complicación fue sometido a una manga gástrica y evolucionó sin

Tabla 1. Características demográficas de la cohorte en general y según tipo de cirugía

\begin{tabular}{|lccc|}
\hline & Manga gástrica & Bypass gástrico & Todos \\
\hline Pacientes (n) & 292 & 36 & 328 \\
\hline Edad promedio (años) & 37 & 45 & 38 \\
\hline Femenino: Masculino (n) & $190: 102$ & $10: 26$ & $200: 128$ \\
IMC preoperatorio & 36,2 & 37,2 & 36,3 \\
\hline Estadía hospitalaria (días) & 2,8 & $7 / 3,4 *$ & 3,2 \\
Presencia de filtración (n) & 1 & 1 & 2 \\
\hline Seguimiento a 3 meses (\%) & $96,9 \%$ & $91,6 \%$ & $96,3 \%$ \\
\hline
\end{tabular}

*3,4 excluyendo paciente outlayer con múltiples complicaciones. 


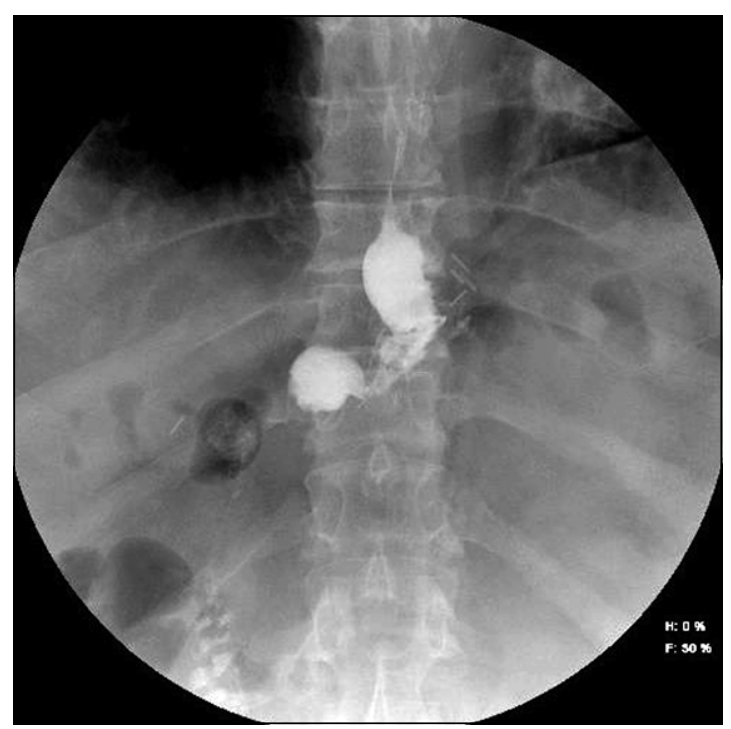

Figura 1. ECTDS realizado al primer día postoperatorio, con cambios postquirúrgicos típicos y sin evidencia de filtración.

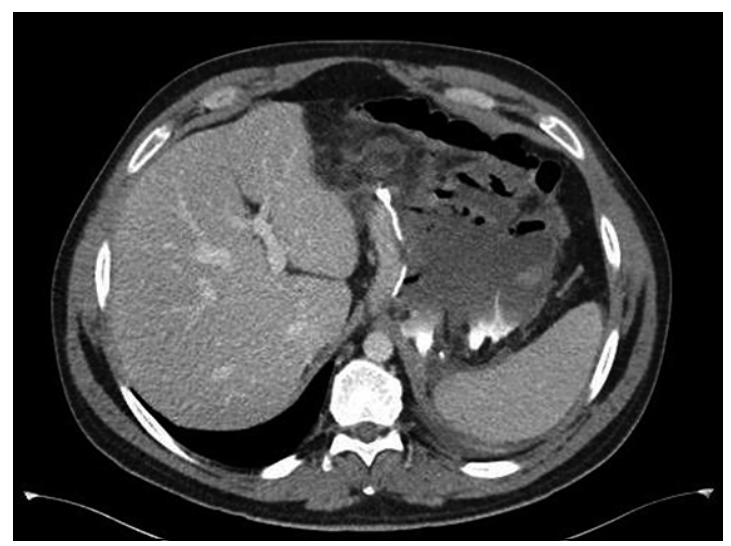

Figura 2. TC de abdomen realizada al sexto día postoperatorio demuestra colección adyacente al remanente gástrico con gas en su interior y presencia de medio de contraste oral.

Tabla 2. Sensibilidad ECTDS post cirugía bariátrica. Evaluación del test diagnóstico: utilidad del ECTDS en la detección de filtración, comparada contra el seguimiento

\begin{tabular}{|llccc|}
\hline \multicolumn{4}{c|}{$\begin{array}{c}\text { Seguimiento } \\
\text { Filtración + Filtración }\end{array}$} & \multicolumn{1}{c|}{ Total } \\
\hline ECTDS & Filtración + & 0 & 0 & 0 \\
& Filtración - & 2 & 306 & 308 \\
Total & 2 & 306 & 308 \\
\hline
\end{tabular}

incidentes con un ECTDS sin signos de complicación (Figura 1). Reingresó al sexto día de postoperatorio, con dolor abdominal y signos clínicos al examen físico de complicación quirúrgica. Por tomografía computada se evidenció la existencia de una colección adyacente al remanente gástrico con gas en su interior y presencia de medio de contraste oral (Figura 2). Se reintervino sin evidencia de filtración macroscópica ni con las pruebas de agua-aire ni azul de metileno. Se instaló un drenaje Babcock, el que dio salida a saliva 2 días después (amilasa 37.570 UI/L), confirmando la filtración, y manejándose con prótesis esófago-intestinal y drenaje percutáneo de manera exitosa. El segundo paciente fue sometido a bypass gástrico y evolucionó con taquicardia, salida de contenido sero-hemático a través del drenaje y febrículas el primer día postoperatorio. El ECTDS sólo evidenció paso lento del medio de contraste. En el segundo día de postoperatorio el paciente evolucionó con shock séptico y se observó salida de contenido intestinal por el drenaje. El intraoperatorio confirmó la presencia de apertura de la sutura del remanente gástrico, que impresionó secundaria a obstrucción del asa biliar en la unión entero-enteral por molde de coágulos, sin obstrucción del asa alimentaria. Se manejó con extracción de coágulos y gastrostomía del remanente. Evolucionó tórpidamente hasta el día postoperatorio número 19 , cuando la tomografía computada de control evidenció una zona de filtración en la anastomosis gastro-yeyunal y una colección adyacente. Se puncionó la colección y se instaló un drenaje percutáneo, con posterior cierre espontáneo de la filtración. Evolucionó con otras complicaciones médicas, no relevantes de señalar en este estudio.

En ambos casos el examen radiológico con contraste no mostró evidencia de filtración postoperatoria (Tabla 2). La sensibilidad para la presencia de filtración del ECTDS en este estudio resultó ser de $0 \%$ y la especificidad de $100 \%$. Considerando la incidencia de filtración de $0,6 \%$, el valor predictivo negativo fue de $99,4 \%$.

\section{Discusión}

Este estudio evidenció una incidencia de filtración similar a la reportada en la literatura internacional, y algo menor que la reportada en un hospital universitario nacional ${ }^{12}$. Esto puede 
deberse a la experiencia adquirida en los últimos años por los centros y cirujanos nacionales, al haber superado la curva de aprendizaje. La evidencia apunta que la ocurrencia de filtración es mayor dentro de los primeros 100 casos de un centro $^{13}$, casuística ampliamente superada en la presente serie.

Por otro lado, la sensibilidad del ECTDS de rutina en el primer día de postoperatorio para la detección de filtración en este estudio fue $0 \%$, aun menor a lo publicado en la literatura (entre 22 y $75 \%)^{2,3,10}$. Esto sería concomitante con la presentación clínica, ya que dentro del primer día postoperatorio sólo se ha presentado $8 \%$ de las filtraciones, siendo más frecuentes la filtración intermedia (entre $5^{\circ}$ y $9^{\circ}$ día postoperatorio), y tardía ( $10^{\circ}$ o más) abarcando $45 \%$ y $33 \%$ de las filtraciones respectivamente ${ }^{12}$.

Por lo tanto, la utilidad de realizar ECTDS de rutina luego de una cirugía bariátrica no parece una indicación necesaria en estos pacientes, dada la baja incidencia de filtración en centros con alta atención de estos pacientes y, en segundo lugar, por la baja sensibilidad del ECTDS en el primer día postoperatorio dada la aparición más tardía de esta complicación. Muy probablemente, el edema de los bordes anastomóticos impide la manifestación radiológica de filtraciones que mayoritariamente son muy pequeñas, lo que se asocia a insuficiente tiempo para dar cuenta de procesos de remodelación tisular inflamatorios.

Vale la pena también plantear dos consideraciones importantes. La primera de ellas se refiere a que en todos estos casos hay una clara clínica en la anamnesis y al examen físico. Como especialistas, que ocupan lugar preponderante en la fase de diagnóstico, corresponde a los radiólogos orientar el estudio con imágenes, pero haciendo prevalecer el examen clínico y luego de ello elegir el método de mejores resultados, y mejor aún si se fundamenta en experiencia local respaldada (investigación de resultados).

La segunda consideración, y no menos importante, se refiere a lo poco costo-efectivo de este examen, dada la baja ocurrencia de la complicación buscada y la discreta sensibilidad del procedimiento, lo que es concordante con la literatura internacional que apunta en contra de su uso rutinario ${ }^{10}$. Finalmente, en los pacientes postoperados con sospecha clínica de filtración, en vez de la realización de ECTDS, el American
College of Radiology sugiere la realización de tomografía computada de abdomen y pelvis con contraste endovenoso ${ }^{14}$.

En conclusión, el ECTDS practicado precozmente y de rutina luego de la cirugía bariátrica laparoscópica no parece ser un examen útil en la búsqueda de filtración en centros con experiencia en cirugía bariátrica.

\section{Referencias}

1. Encuesta Nacional de Salud 2009-2010, MINSAL. Consultado online el 14 de agosto de 2014 en web.minsal.cl/ portal/url/.../bcb03d7bc28b64dfe040010165012d23.pdf.

2. Doraiswamy A, Rasmussen JJ, Pierce J, Fuller W, Ali MR. The utility of routine postoperative upper GI series following laparoscopic gastric bypass. Surg Endosc 2007; 21 (12): 2159-62.

3. Madan AK, Stoecklein HH, Ternovits CA, Tichansky DS, Phillips JC. Predictive value of upper gastrointestinal studies versus clinical signs for gastrointestinal leaks after laparoscopic gastric bypass. Surg Endosc 2007; 21 (2): 194-6.

4. Singh R, Fisher BL. Sensitivity and specificity of postoperative upper GI series following gastric bypass. Obes Surg 2003; 13: 73- 5.

5. Brockmeyer JR, Simon TE, Jacob RK, Husain F, Choi Y. Upper gastrointestinal swallow study following bariatric surgery: institutional review and review of the literature. Obes Surg 2012; 22 (7): 1039-43.

6. Dallal RM, Bailey L, Nahmias N. Back to basics-clinical diagnosis in bariatric surgery. Routine drains and upper GI series are unnecessary. Surg Endosc 2007; 21 (12): 2268-71.

7. Mittermair R, Sucher R, Perathoner A, Wykypiel H. Routine upper gastrointestinal swallow studies after laparoscopic sleeve gastrectomyare unnecessary. Am J Surg 2014; 207 (6): 897-901.

8. Márquez MF, Ayza MF, Lozano RB, Morales M del M, Díez JM, Poujoulet RB. Gastric leak after laparoscopic sleeve gastrectomy. Obes Surg 2010; 20: 1306-11. doi:10.1007/s11695-010-0219-7.

9. Bertelson NL, Myers JA. Routine postoperative upper gastrointestinal fluoroscopy is unnecessary after laparoscopic adjustable gastric band placement. Surg Endosc 2010; 24: 2188-91.

10. Schiesser M, Guber J, Wildi S, Guber I, Weber M, Muller MK. Utility of routine versus selective upper gastrointestinal series to detect anastomotic leaks after laparoscopic gastric bypass. Obes Surg 2011; 21 (8): 1238-42. 
11. de Grazia KJA, Godoy ZM, Torrealba AI, Guzmán GM, Csendes P, Csendes JA. Estudio baritado del tubo digestivo alto en pacientes postoperados de cirugía bariátrica: Hallazgos normales y patológicos. Rev Chil Radiol 2013; 19 (2): 53-9.

12. Burgos AM. Filtraciones en bypass gástrico resectivo por laparotomía en obesidad mórbida. Rev Chil Cir 2012; 64: 52-62.

13. Carter JT, Tafreshian S, Campos GM, Tiwari U, Herbella
F, Cello JP, et al. Routine upper GI series after gastric bypass does not reliably identify anastomotic leaks or predict stricture formation. Surg Endosc 2007; 21 (12): 2172-7.

14. Yaghmai V, Rosen MP, Lalani T, Baker ME, Blake MA, et al. ACR Appropriateness Criteria ${ }^{\circledR}$ Acute Abdominal Pain Fever Suspected Abdominal Abscess. Available at https://acsearch.acr.org/docs/69467/Narrative/. American College of Radiology. Accessed Mar 30, 2014. 